\title{
QUALIDADE DE VIDA, DEPRESSÃO E ANSIEDADE EM ENFERMEIROS E TÉCNICOS DE ENFERMAGEM DE UM HOSPITAL
}

Quality of life, depression and anxiety in nurses and nurse technicians from a hospital

Calidad de vida, depresión y ansiedad en enfermeras y técnicos de enfermería em um hospital

Bárbara do Nascimento Lopes Pessoa ${ }^{*}{ }^{1}$, Leilane Camila Ferreira de Lima Francisco ${ }^{3}$, Helcimara Martins Golçalves Ramos², Yanna Cristina Moraes Lira Nascimento ${ }^{1}$, Verônica de Medeiros Alves ${ }^{3}$

${ }^{1}$ Curso de Graduação em Enfermagem, Universidade Federal de Alagoas, Maceió-AL, Brasil.

${ }^{2}$ Hospital Escola Portugal Ramalho, Universidade Estadual de Ciências da Saúde de Alagoas, Maceió-AL, Brasil.

${ }^{3}$ Programa de Pós-Graduação em Enfermagem (Mestrado), Universidade Federal de Alagoas, Maceió-AL, Brasil.

*Correspondência: Rua Liberato Mitchel, $n^{\circ}$ 111, Tabuleiro dos Martins. Maceió-AL. CEP: 57081-105.e-mail barbaralopespessoa@hotmail.com.

\section{Artigo recebido em 01/12/2020 aprovado em 27/04/2021 publicado em 20/10/2021.}

\section{RESUMO}

Entre os trabalhadores da saúde, os profissionais de enfermagem estão no grupo dos mais propensos aos problemas de saúde mental, porque lidam com o sofrimento humano e enfrentam sobrecarga de trabalho. Diante disso, este estudo tem como objetivo avaliar a qualidade de vida, depressão e ansiedade em enfermeiros e técnicos de enfermagem de um hospital psiquiátrico. Trata-se de um estudo quantitativo, descritivo e transversal realizado na cidade de Maceió-AL entre outubro de 2018 e janeiro de 2019, sendo aprovado pelo Comitê de Ética em Pesquisa da Universidade Federal de Alagoas. Foram entrevistados 75 profissionais de enfermagem, sendo 65 (86,7\%) técnicos de enfermagem e $10(13,3 \%)$ enfermeiros. 51 (68\%) apresentaram risco para depressão, $64(85,3 \%)$ tinham ansiedade traço moderada e $63(84 \%)$ ansiedade estado moderada. Obtiveram-se as médias de qualidade de vida: domínio físico $(55,0)$, psicológico $(73,2)$, relação social $(65,3)$, ambiente $(57,7)$ e qualidade de vida geral $(62,3)$. A comorbidade ansiedade depressão apresentou menor média em todos os domínios. Evidenciou-se que os profissionais de enfermagem apresentaram qualidade de vida pouco comprometida, risco para depressão e níveis moderados de ansiedade.

Palavras-chave: Saúde mental. Qualidade de vida. Ansiedade. Depressão. Enfermagem.

\section{ABSTRACT}

Among health workers, nursing professionals are among the group most prone to mental health problems, because they deal with human suffering and face work overload. Therefore, this study aims to evaluate the quality of life, depression and anxiety in nurses and nursing technicians in a psychiatric hospital. This is a quantitative, descriptive and cross-sectional study conducted in the city of Maceio-AL between October 2018 and January 2019, being approved by the Research Ethics Committee of the Federal University of Alagoas. 75 professionals were interviewed, $10(13.3 \%)$ nurses and 65 (86.7\%) nursing technicians. 51 (68\%) were at risk for depression, 64 (85.3\%) had 
moderate trait anxiety and 63 (84\%) moderate state anxiety. The means of quality of life were obtained: physical domain (55.0), psychological (73.2), social relationship (65.3), environment (57.7) and general quality of life (62.3). The anxiety depression comorbidity showed a lower average in all domains. It was evidenced that nursing professionals had little impaired quality of life, risk for depression and moderate levels of anxiety.

Keywords: Mental health. Quality of life. Anxiety. Depression. Nursing.

\section{RESUMEN}

Entre los trabajadores de la salud, los profesionales de enfermería se encuentran entre el grupo más propenso a los problemas de salud mental, porque enfrentan el sufrimiento humano y enfrentan una sobrecarga de trabajo. Por tanto, este estudio tiene como objetivo evaluar la calidad de vida, la depresión y la ansiedad en enfermeros y técnicos de enfermería de un hospital psiquiátrico. Se trata de un estudio cuantitativo, descriptivo y transversal realizado en la ciudad de Maceió-AL entre octubre de 2018 y enero de 2019, siendo aprobado por el Comité de Ética en Investigación de la Universidad Federal de Alagoas. Se entrevistaron 75 profesionales, 10 (13,3\%) enfermeros y 65 (86,7\%) técnicos de enfermería. 51 (68\%) tenían riesgo de depresión, 64 (85,3\%) tenían un rasgo de ansiedad moderado y 63 (84\%) un estado de ansiedad moderado. Se obtuvo la calidad de vida promedio: dominio físico (55,0), psicológico (73,2), relación social (65,3), medio ambiente (57,7) y calidad de vida general (62,3). La comorbilidad ansiedad-depresión mostró un promedio más bajo en todos los dominios. Se evidenció que los profesionales de enfermería tenían poca calidad de vida comprometida, riesgo de depresión y niveles moderados de ansiedad.

Descriptores: Salud mental. Calidad de vida. Ansiedad. Depresión. Enfermería.

\section{INTRODUÇÃO}

O trabalho ocupa uma maior parcela da vida de cada indivíduo e sua satisfação está associada a um maior bem-estar, realização pessoal e valorização profissional (VIEIRA et al., 2018). Assim, a qualidade de vida é conceituada de forma ampla e abrange como o indivíduo a percebe no contexto cultural e de valores, por meio da relação de suas metas, expectativas, padrões e preocupações (ARAÚJO et al., 2018). Além disso, ela se relaciona com os aspectos físicos, psicológicos, sociais, intelectuais e econômicos, baseando-se em princípios fundamentais, dos quais se pode destacar a capacidade funcional, o nível socioeconômico e a satisfação (ARAÚJO et al., 2018).

Desse modo, no processo de trabalho dos profissionais de enfermagem, fatores como as cargas laborais, as condições de trabalho e os riscos ocupacionais somam-se a oportunidades insuficientes de lazer, reduzindo o tempo dedicado ao autocuidado e às relações sociais. Esse cenário é associado às características ocupacionais, como o regime de plantões, aos múltiplos vínculos, ao intenso ritmo laboral, acompanhadas por cargas horárias extensas.
Diante disso, cria-se um contexto favorável para o estresse, desgaste, adoecimento e comprometendo a qualidade de vida (VIEIRA et al., 2018).

Trabalhar em setores que demandam mais atenção e com dinâmica mais intensa de trabalho favorece o aumento do risco para transtornos mentais, devido a características dos eventos estressores que ocorrem nesse ambiente (OLIVEIRA et al., 2019).

Entre os trabalhadores da saúde, os profissionais de enfermagem estão no grupo dos mais propensos aos problemas de saúde mental, porque lidam com o sofrimento humano, a dor, a alegria, tristeza e necessitam ofertar ajuda àqueles que necessitam de seus cuidados (SILVA et al., 2015).

No contexto de alterações psicológicas na força de trabalho, a depressão e ansiedade são preocupantes devido à alta prevalência e altos custos. Os transtornos depressivos incluem outros subtipos, sendo o transtorno depressivo maior o representante clássico desse grupo. São caracterizados por alterações do humor, que possuem como característica em comum, a presença de humor triste (APA, 2014). 
Estima-se que 15 a $25 \%$ da população do mundo sofram com o transtorno depressivo, ou seja, a cada quatro pessoas, uma apresentará os sintomas de depressão, necessitando de tratamento pelo menos uma vez na vida. No Brasil, estatísticas apontam que aproximadamente 24 a 30 milhões de pessoas já apresentaram, apresentam ou irão apresentar pelo menos um episódio depressivo ao longo da vida (SANTOS et al., 2018).

O transtorno de ansiedade se difere da ansiedade e do medo adaptativos que são inerentes à vida de qualquer indivíduo. Quando esse estado de ansiedade se cronifica, torna-se excessivo e/ou persiste por longos períodos de tempo impossibilitando o indivíduo, é caracterizado o transtorno de ansiedade (APA, 2014).

Sendo assim, cabe a este estudo responder à seguinte questão: "Os enfermeiros e técnicos de enfermagem de um hospital psiquiátrico apresentam qualidade de vida comprometida, depressão e ansiedade?". Seu objetivo foi avaliar a qualidade de vida, depressão e ansiedade em enfermeiros e técnicos de enfermagem de um hospital psiquiátrico localizado na cidade de Maceió - Alagoas.

\section{MATERIAIS E MÉTODOS}

Trata-se de um estudo quantitativo, descritivo e transversal. A amostra planejada para esta pesquisa foi, inicialmente, por conveniência, na qual todos os trabalhadores de enfermagem foram abordados para a entrevista. Um dos setores veio a ser desanexado e passou a realizar as suas atividades em outro prédio. Com isso, a população de profissionais atuantes no hospital passou a ser de 16 enfermeiros e 85 técnicos de enfermagem, totalizando 101 profissionais. Tendo em vista que nem todos aceitaram participar do estudo, a amostra final foi composta então por 75 trabalhadores da enfermagem, dos quais 10 eram enfermeiros e 65 eram técnicos de enfermagem.

Foram utilizados quatro instrumentos: Questionário de Identificação, Escala de depressão do Center for Epidemiologic Studies - CES-D, Inventário de Ansiedade Traço-Estado - IDATE e o questionário sobre qualidade de vida (WHOQOL - bref).

A validação da escala CES-D foi feita por Radloff (1977) e foi validada no Brasil por Dartiu Xavier da Silva \& Miguel R. Jorge (1997). Esta escala avalia a frequência de ocorrência de 20 indicadores do quadro depressivo, com uma estrutura de quatro fatores: afetos positivos, depressão, aspectos somáticos/atividade reduzida e problemas interpessoais, buscando essas dimensões abranger os principais aspectos afetivos, cognitivos, somáticos e comportamentais da depressão (HAUCK FILHO, 2011).

O Inventário de Ansiedade Traço-Estado (IDATE) foi desenvolvido por Spielberger, Gorsuch e Lushene (1970), traduzido e adaptado para o Brasil por Biaggio (Biaggio \& Natalício 1979). O IDATE apresenta uma escala que avalia a ansiedade enquanto estado (IDATE-E) e outra que avalia a ansiedade enquanto traço (IDATE-T). É uma escala de autoaplicável que pode ser utilizado individualmente ou em grupo (CAPITÃO; TELLO, 2004).

O WHOQOL-bref, uma versão abreviada do WHOQOL-100, desenvolvido e recomendado pela Organização Mundial da Saúde (OMS), composto por 26 questões, sendo 2 referentes à qualidade de vida geral e 24 questões para avaliar os domínios de saúde física e psicológica, as relações sociais e o meio ambiente no qual o participante vive (ALMEIDABRASIL et al., 2017).

As variáveis de interesse deste estudo foram as seguintes: sexo, estado civil, tem filhos, local onde 
reside, tempo gasto da residência até o trabalho, tempo que trabalha no hospital, categoria profissional, tem qualificação profissional, tipo de qualificação profissional, carga horária de trabalho semanal, trabalha em outro local, trabalha como enfermeiro ou técnico de enfermagem, turnos de trabalho, risco para depressão e ansiedade, qualidade de vida. Foi realizado como tratamento estatístico frequência absoluta e relativa, média e desvio padrão, sendo utilizado o software SPSS, versão 20, para organização e formulação de tabelas resultantes das comparações necessárias entre as variáveis utilizadas.

O critério de inclusão para participar da pesquisa consistiu em ser enfermeiro ou técnico de enfermagem no exercício de sua função no Hospital Psiquiátrico Estadual localizado na cidade de Maceió Alagoas. Para critérios de exclusão, foram considerados enfermeiros e técnicos de enfermagem que se encontravam afastados do trabalho por licença médica, licença maternidade, aposentados, exonerados ou por qualquer outro motivo que os levem a estar afastados do serviço e profissionais cedidos para outra instituição. Os enfermeiros e técnicos de enfermagem foram convidados a participar da pesquisa e, ao concordarem, assinaram o Termo de Consentimento Livre e Esclarecido (TCLE).

O projeto foi aprovado pelo Comitê de Ética em Pesquisa da Universidade Federal de Alagoas (UFAL) sob o parecer n. 2.857.915, em 31 de agosto de 2018. Todos os participantes foram informados sobre as etapas do estudo.

\section{RESULTADOS E DISCUSSÃO}

Foram entrevistados $65(86,7 \%)$ técnicos de enfermagem e $10(13,3 \%)$ enfermeiros. No momento em que houve a pesquisa, 16 enfermeiros e 85 técnicos de enfermagem trabalhavam no hospital. Eles afirmaram possuir alguma qualificação profissional (26
- 34,7\%), destacando-se a especialização (24 - 32\%) (Tabela 1).

No que se diz respeito ao sexo, a maioria dos profissionais era do sexo feminino (59-78,7\%). Desses profissionais, os solteiros (28 - 37,3\%) e casados (28 $37,3 \%)$ eram maioria e $55(73,3 \%)$ tinham filhos (Tabela 1). Sobre o local de residência, 59 (78,7\%) moravam em Maceió, 33 (40\%) levavam entre trinta minutos a uma hora para chegarem ao local de trabalho, enquanto $25(33,3 \%)$ levavam menos de trinta minutos. $64(85,3 \%)$ trabalhavam no hospital há mais de um ano (Tabela 1).

A carga horária de trabalho predominante era de 30 horas semanais (69 - 92\%). Entre os profissionais, $43(57,3 \%)$ trabalhavam em outro local. Quanto à função laboral exercida neste outro local, 39 (52\%) exerciam a função de enfermeiro ou técnico e 4 $(5,3 \%)$ realizavam outro tipo de trabalho. A escala de trabalho implantada no hospital era de 12/12 horas de serviço, podendo ter algumas exceções. A maioria dos profissionais $(63$ - 84\%) trabalhava nos horários matutino, vespertino e noturno (Tabela 1).

No que diz respeito ao risco para depressão, os valores obtidos evidenciaram que a maioria dos profissionais (51 - 68\%) possuíam esse risco (Tabela 2). Na avaliação de ansiedade traço, os profissionais apresentaram ansiedade moderada $(64-85,3 \%)$ e ansiedade leve (10 - 13,3\%). Na ansiedade estado, 63 (84\%) apresentaram ansiedade moderada, 10 (13,3\%) ansiedade leve e um $(1,3 \%)$ ansiedade alta. Ao vincular os dados de ansiedade traço-estado, 50 (66,7\%) apresentaram ansiedade moderada e 24 (32,7\%) ansiedade alta (Tabela 2).

Os dados ansiedade e depressão foram cruzados com a variável sexo, demonstrando uma maior frequência de ansiedade traço e estado moderados nas mulheres (Tabela 2). 
Tabela 1. Dados gerais de enfermeiros e técnicos de enfermagem do Hospital Escola Portugal Ramalho, Maceió, Alagoas, 2018. N=75.

\begin{tabular}{|c|c|c|c|}
\hline \multicolumn{2}{|c|}{ Dados gerais } & $\mathbf{N}$ & $\%$ \\
\hline \multirow{2}{*}{ Sexo } & Masculino & 16 & 21,3 \\
\hline & Feminino & 59 & 78,7 \\
\hline \multirow{5}{*}{ Estado civil } & Solteiro & 28 & 37,3 \\
\hline & Casado & 28 & 37,3 \\
\hline & União estável & 3 & 4 \\
\hline & Viúvo(a) & 13 & 17,3 \\
\hline & Divorciado & 3 & 4 \\
\hline \multirow{2}{*}{ Tem filhos } & Sim & 55 & 73,3 \\
\hline & Não & 20 & 26,7 \\
\hline \multirow{2}{*}{ Local onde reside } & Maceió & 59 & 78,7 \\
\hline & Outra cidade & 16 & 21,3 \\
\hline \multirow{3}{*}{$\begin{array}{c}\text { Tempo gasto da residência } \\
\text { até o trabalho }\end{array}$} & $<30 \min$ & 25 & 33,3 \\
\hline & Entre $30 \mathrm{~min}$ e 1 hora & 33 & 40 \\
\hline & $>1$ hora & 17 & 22,7 \\
\hline \multirow{2}{*}{$\begin{array}{c}\text { Tempo que trabalha no } \\
\text { hospital }\end{array}$} & $<1$ ano & 11 & 14,7 \\
\hline & $>1$ ano & 64 & 85,3 \\
\hline \multirow{2}{*}{ Categoria profissional } & Técnico de enfermagem & 65 & 86,7 \\
\hline & Enfermeiro & 10 & 13,3 \\
\hline \multirow{2}{*}{$\begin{array}{l}\text { Tem qualificação } \\
\text { profissional }\end{array}$} & Sim & 26 & 34,7 \\
\hline & Não & 49 & 65,3 \\
\hline \multirow{3}{*}{$\begin{array}{c}\text { Tipo de qualificação } \\
\text { profissional }\end{array}$} & Especialização & 24 & 32 \\
\hline & Estudante de mestrado & 1 & 1,3 \\
\hline & Mestre & 1 & 1,3 \\
\hline \multirow{2}{*}{$\begin{array}{c}\text { Carga horária de trabalho } \\
\text { semanal }\end{array}$} & 30 horas & 69 & 92 \\
\hline & 40 horas & 6 & 8 \\
\hline \multirow{3}{*}{ Trabalha em outro local } & Sim & 43 & 57,3 \\
\hline & Não & 30 & 40 \\
\hline & Sem resposta & 2 & 2,7 \\
\hline \multirow{2}{*}{$\begin{array}{c}\text { Como enfermeiro/técnico } \\
\text { de enfermagem? }\end{array}$} & Sim & 39 & 52 \\
\hline & Não & 4 & 5,3 \\
\hline \multirow{4}{*}{ Turnos de trabalho } & Matutino & 3 & 4 \\
\hline & Noturno & 5 & 6,7 \\
\hline & Matutino, vespertino & 4 & 5,3 \\
\hline & $\begin{array}{c}\text { Matutino, vespertino e } \\
\text { noturno }\end{array}$ & 63 & 84 \\
\hline
\end{tabular}

Fonte: Dados da pesquisa, 2018 
Tabela 2. Risco para depressão e ansiedade em enfermeiros e técnicos de enfermagem do Hospital Escola Portugal Ramalho, Maceió, Alagoas, 2018. N=75.

\begin{tabular}{|c|c|c|c|c|c|}
\hline Transtorno & Classificação & \multicolumn{2}{|c|}{$\mathbf{N}$} & \multicolumn{2}{|c|}{$\%$} \\
\hline \multirow{2}{*}{ Depressão } & Com risco & \multicolumn{2}{|c|}{51} & \multicolumn{2}{|c|}{68} \\
\hline & Sem risco & \multicolumn{2}{|c|}{22} & \multicolumn{2}{|c|}{29,3} \\
\hline \multirow{2}{*}{ Ansiedade traço } & Baixa & \multicolumn{2}{|c|}{10} & \multicolumn{2}{|c|}{13,3} \\
\hline & Moderada & \multicolumn{2}{|c|}{64} & \multicolumn{2}{|c|}{85,3} \\
\hline \multirow{3}{*}{ Ansiedade estado } & Baixa & \multicolumn{2}{|c|}{10} & \multicolumn{2}{|c|}{13,3} \\
\hline & Moderada & \multicolumn{2}{|c|}{63} & \multicolumn{2}{|c|}{84} \\
\hline & Alta & \multicolumn{2}{|c|}{1} & \multicolumn{2}{|c|}{1,3} \\
\hline \multirow{2}{*}{ Ansiedade total } & Moderada & \multicolumn{2}{|c|}{50} & \multicolumn{2}{|c|}{66,7} \\
\hline & Alta & \multicolumn{2}{|c|}{24} & \multicolumn{2}{|c|}{32,7} \\
\hline \multirow{3}{*}{ Transtorno } & \multirow{3}{*}{ Classificação } & \multicolumn{4}{|c|}{ Sexo } \\
\hline & & \multicolumn{2}{|c|}{ Feminino } & \multicolumn{2}{|c|}{ Masculino } \\
\hline & & $\mathbf{N}$ & $\%$ & $\mathbf{N}$ & $\%$ \\
\hline \multirow{2}{*}{ Ansiedade traço } & \multirow{2}{*}{$\begin{array}{c}\text { Baixa } \\
\text { Moderada }\end{array}$} & 7 & 12 & 3 & 18,8 \\
\hline & & 51 & 87,9 & 13 & 81,3 \\
\hline \multirow{3}{*}{ Ansiedade estado } & Baixa & 8 & 13,8 & 2 & 12,5 \\
\hline & Moderada & 49 & 84,5 & 14 & 87,5 \\
\hline & Alta & 1 & 1,7 & . & . \\
\hline \multirow{2}{*}{ Depressão } & Com risco & 39 & 68,4 & 12 & 75 \\
\hline & Sem risco & 18 & 31,6 & 4 & 25 \\
\hline \multirow{2}{*}{ Depressão e ansiedade } & Sim & 12 & 25,5 & 2 & 14,3 \\
\hline & Não & 47 & 79,7 & 14 & 87,5 \\
\hline
\end{tabular}

Fonte: Dados da pesquisa, 2018.

Ao analisar as médias dos dados quanto à qualidade de vida e seus domínios coletados, obtivemos os seguintes valores: menor média no domínio físico $(55,0 \pm 18,3)$ e maior no domínio psicológico $(73,2 \pm 14,6)$, apresentando uma média de qualidade de vida geral de 62,3 $( \pm 26,1)$ (Tabela 3$)$.

Ao cruzar os dados de qualidade de vida e o risco para depressão, encontrou-se menor média no domínio físico $(50,0 \pm 14,7)$ e maior no psicológico $(75,7 \pm 15)$, apresentando uma média de qualidade de vida de 65,9 ( \pm 19$)$ (Tabela 3$)$.

Considerando a ansiedade traço, a menor média de qualidade de vida foi no domínio meio ambiente para ansiedade traço leve $(55 \pm 21,0)$ e moderada $(55 \pm 18,2)$ e a maior média no domínio psicológico $(73,5 \pm 14,9)$ para ansiedade traço moderada. Em ansiedade estado, a menor média de qualidade de vida encontrada foi no domínio meio ambiente $(51,6 \pm 20,7)$ para ansiedade estado leve e a maior média no domínio psicológico (100) em ansiedade estado alta (Tabela 3 ).

Os dados de depressão e ansiedade foram cruzados com o de qualidade de vida e identificou-se que nos profissionais que apresentavam como comorbidade, o risco para depressão e ansiedade moderada e alta obtiveram menor média no domínio físico $(46,4 \pm 14,3)$ e maior média no domínio psicológico $(71,4 \pm 16,8)$ (Tabela 3$)$. 
Tabela 3. Depressão, ansiedade e qualidade de vida em enfermeiros e técnicos de enfermagem do Hospital Escola Portugal Ramalho, Maceió, Alagoas, 2018. N=75.

\begin{tabular}{|c|c|c|c|c|c|c|}
\hline \multirow{2}{*}{\multicolumn{2}{|c|}{ Qualidade de vida }} & \multicolumn{4}{|c|}{ Domínios de qualidade de vida } & \multirow{2}{*}{$\begin{array}{c}\text { Qualidade de vida } \\
\text { geral }\end{array}$} \\
\hline & & Físico & Psicológico & $\begin{array}{l}\text { Relações } \\
\text { sociais }\end{array}$ & Meio ambiente & \\
\hline \multicolumn{2}{|c|}{ Média } & 55 & 73,2 & 65,3 & 57,7 & 62,3 \\
\hline \multicolumn{2}{|c|}{ Desvio padrão } & 18,3 & 14,6 & 18,3 & 16,5 & 26,1 \\
\hline \multirow{2}{*}{\multicolumn{2}{|c|}{ Depressão }} & \multicolumn{4}{|c|}{ Domínios de qualidade de vida } & Oualidade de vida \\
\hline & & Físico & Psicológico & $\begin{array}{l}\text { Relações } \\
\text { sociais }\end{array}$ & Meio ambiente & geral \\
\hline \multirow[b]{2}{*}{ Sem risco } & Média & 56,7 & 72,0 & 63,1 & 56,8 & 62,7 \\
\hline & $\begin{array}{l}\text { Desvio } \\
\text { padrão }\end{array}$ & 19,5 & 14,5 & 18,0 & 18,2 & 26,3 \\
\hline \multirow{2}{*}{ Com risco } & Média & 50,0 & 75,7 & 69,3 & 58,9 & 65,9 \\
\hline & $\begin{array}{l}\text { Desvio } \\
\text { padrão }\end{array}$ & 14,7 & 15,0 & 18,6 & 12,8 & 19,0 \\
\hline \multirow{2}{*}{\multicolumn{2}{|c|}{ Ansiedade traço }} & \multicolumn{4}{|c|}{ Domínios de qualidade de vida } & Oualidade de vida \\
\hline & & Físico & Psicológico & $\begin{array}{c}\text { Relações } \\
\text { sociais }\end{array}$ & Meio ambiente & geral \\
\hline \multirow[b]{2}{*}{ Baixa } & Média & 55,0 & 71,7 & 59,2 & 59,1 & 57,5 \\
\hline & $\begin{array}{l}\text { Desvio } \\
\text { padrão }\end{array}$ & 21,0 & 13,1 & 25,6 & 16,8 & 27,8 \\
\hline \multirow[b]{2}{*}{ Moderada } & Média & 55,0 & 73,5 & 66,3 & 57,3 & 63,3 \\
\hline & $\begin{array}{l}\text { Desvio } \\
\text { padrão }\end{array}$ & 18,2 & 14,9 & 17,1 & 16,7 & 26,1 \\
\hline \multirow{2}{*}{\multicolumn{2}{|c|}{ Ansiedade estado }} & \multicolumn{4}{|c|}{ Domínios de qualidade de vida } & Oualidade de vida \\
\hline & & Físico & Psicológico & $\begin{array}{l}\text { Relações } \\
\text { sociais }\end{array}$ & Meio ambiente & $\begin{array}{l}\text { Qualiaade de vida } \\
\text { geral }\end{array}$ \\
\hline \multirow[t]{2}{*}{ Baixa } & Média & 62,5 & 75,4 & 70,0 & 51,6 & 57,5 \\
\hline & $\begin{array}{l}\text { Desvio } \\
\text { padrão }\end{array}$ & 17,3 & 7,7 & 16,8 & 20,7 & 24,4 \\
\hline \multirow[t]{2}{*}{ Moderada } & Média & 53,8 & 72,5 & 64,5 & 58,2 & 63,1 \\
\hline & $\begin{array}{l}\text { Desvio } \\
\text { padrão }\end{array}$ & 18,6 & 15,2 & 18,8 & 15,8 & 26,7 \\
\hline \multirow[t]{2}{*}{ Alta } & Média & 57,1 & 100 & 66,7 & 75,0 & 75 \\
\hline & $\begin{array}{l}\text { Desvio } \\
\text { padrão }\end{array}$ & $\cdot$ & $\cdot$ & . & . & $\cdot$ \\
\hline \multirow{2}{*}{\multicolumn{2}{|c|}{ Depressão e ansiedade }} & \multicolumn{4}{|c|}{ Domínios de qualidade de vida } & Oualidade de vida \\
\hline & & Físico & Psicológico & $\begin{array}{l}\text { Relações } \\
\text { sociais }\end{array}$ & Meio ambiente & $\begin{array}{l}\text { Qualidade de vida } \\
\text { geral }\end{array}$ \\
\hline \multirow[b]{2}{*}{ Sim } & Média & 46,4 & 71,4 & 64,9 & 58,9 & 59,8 \\
\hline & $\begin{array}{l}\text { Desvio } \\
\text { padrão }\end{array}$ & 14,3 & 16,8 & 21,5 & 13,9 & 20,3 \\
\hline \multirow[b]{2}{*}{ Não } & Média & 57,0 & 73,6 & 65,4 & 57,4 & 62,9 \\
\hline & $\begin{array}{l}\text { Desvio } \\
\text { padrão }\end{array}$ & 18,7 & 14,1 & 17,7 & 17,1 & 27,3 \\
\hline
\end{tabular}

Fonte: Dados da pesquisa, 2018.

Estudo realizado em João Pessoa, Brasil, com enfermeiros e técnicos de enfermagem identificou prevalência maior de profissionais do sexo feminino
(43-89,5\%) (COSTA et al., 2017), semelhante ao que se encontrou na presente pesquisa. 
Desse modo, a predominância do sexo feminino na categoria de enfermagem, apresenta também implicações relevantes no que concerne ao fator sobrecarga de trabalho. Trabalhadoras do sexo feminino precisam conciliar a vida profissional com as atividades domésticas e da família, o que pode ocasionar altos níveis de estresse, comprometer sua qualidade de vida e saúde mental (COSTA, 2018).

Levando em consideração as variáveis idade, estado civil e tempo de trabalho, é importante observar um estudo no qual revelou que o risco para desenvolver depressão aumentou em 13\% a cada um ano de idade aumentado e $16 \%$ para cada ano de trabalho na enfermagem. Identificou-se ainda que enfermeiras solteiras e divorciadas/viúvas foram 10,3 e 10,2 vezes mais propensas à depressão do que as casadas (TSARAS, 2018).

Estudo semelhante quanto a carga horária trabalhada foi realizado com profissionais de enfermagem em três hospitais gerais públicos e universitários de São Paulo, o qual identificou que a maioria $(766$ - 78,97\%) trabalhava com carga horária semanal entre $21 \mathrm{a} 40 \mathrm{~h}$. Vale lembrar que este mesmo estudo identificou que a carga horária, o número de vínculos empregatícios diante dos baixos salários e as más condições no ambiente laboral deixam evidente quão sobrecarregada esta profissão se encontra (GUIMARÃES; FELLI, 2016). Isso corrobora com os achados evidenciados na atual pesquisa.

As variáveis "trabalha em outro local" e "carga horária" são relevantes, pois o acúmulo de empregos levando ao cumprimento de duplas ou triplas jornadas de trabalho interferem diretamente na qualidade de vida e na saúde (SOUZA et al., 2018). Além disso, por presenciarem diariamente e até mesmo serem alvo de violência advinda de pacientes e familiares ou colegas de trabalho, isso torna essa classe profissional ainda mais suscetível a danos à sua saúde (TSUKAMOTO et al., 2019).
Pesquisa realizada com o objetivo de avaliar a prevalência e os fatores de risco para o desenvolvimento de transtornos mentais em uma amostra de 102 enfermeiras australianas identificou que as taxas de depressão, ansiedade e estresse foram de $32,4 \%, 41,2 \%$ e $41,2 \%$, respectivamente (MARAHAJ; LEES; LAL, 2019). Como o atual estudo identificou um risco para depressão de 68\%, comparando com o estudo supracitado, vê-se quão preocupante estava a saúde mental desses profissionais e a necessidade de intervenção precoce, objetivando uma melhoria na qualidade de vida e, consequentemente, na saúde mental.

O presente estudo revelou frequências elevadas para ansiedade traço $(58,3 \%)$ e estado (84\%) moderada. Isso corrobora com um estudo realizado com a equipe de enfermagem de um hospital de São Paulo, que identificou que o grupo apresentou níveis moderados de ansiedade-estado $(49,7 \pm 9,0)$, ansiedade traço $(45,7 \pm 9,5)$ (KUREBAYASHI et al., 2017).

Estudo realizado no Mato Grosso do Sul com 16 enfermeiros identificou sintomas de ansiedade e estresse decorrentes das atividades laborais, sendo eles cefaleia, irritabilidade, perda da concentração, fadiga, alterações do sono, alterações de apetite, comer em excesso para aliviar o estresse e ansiedade, preocupações excessivas, perda de apetite, sensação de desgaste físico constante, problemas de memória e mal-estar generalizado sem causa específica (LEMES et al., 2015).

Ao analisar o risco para depressão por sexo, os dados obtidos foram de $25,5 \%$ no sexo feminino e $14,3 \%$ no masculino, o que coincide com uma pesquisa que buscou analisar os fatores associados à depressão entre homens e mulheres e identificou que este foi mais presente nas mulheres $(33,3 \%)$, enquanto que nos homens a prevalência foi de 12,9\% (SANTOS; BARROS; ANDREOLI, 2019). As explicações para esta prevalência incluem que o gênero feminino é 
muito singular e a saúde mental dessas mulheres sofre interferência de todo um contexto, seja ambiental, social, cultural, econômico e até mesmo da saúde reprodutiva (PARREIRA et al., 2017).

Um estudo que buscou avaliar os fatores associados à qualidade de vida em enfermeiras intensivistas identificou as seguintes médias de acordo com os domínios: físico $(63,04)$, psicológico $(65,42)$, relações sociais $(62,71)$, meio ambiente $(52,73)$, sendo o último domínio aquele que obteve menor média de qualidade de vida (SOUZA et al., 2018). No atual estudo, obteve-se menor média no domínio físico, o que reflete na fragilidade quanto à presença de dor ou desconforto, dependência de medicações, satisfação com o sono, capacidade para trabalhar e atividade de vida diária.

Ao relacionar o risco para depressão, ansiedade e o questionário de qualidade de vida, as médias dos domínios foram maiores nos classificados com risco para depressão, com exceção do domínio físico $(50,0)$; e maiores entre aquelas com ansiedade traço estado moderada e alta, com exceção do domínio meio ambiente $(57,3)$. Estudo aponta uma correlação negativa $(r=-0,38$ domínio físico; $r=-0,46$ domínio psicológico; $r=-0,50$ domínio relações sociais e $r=-$ 0,44 domínio meio ambiente) entre o escore do Inventário de Depressão de Beck (IDB) e os domínios do WHOQOL-bref (RIOS et al., 2010), dados contrários ao obtidos no presente estudo.

Quanto à qualidade de vida dos participantes, existe a possibilidade de os mesmos haverem optado por transmitir um caráter positivo nas suas respostas, mesmo sabendo do resguardo sigiloso e ético dos seus dados

As limitações deste estudo estão relacionadas ao fato de que o mesmo não conseguiu realizar as entrevistas com todos os profissionais da enfermagem desse hospital.

\section{CONCLUSÃO}

Apesar da existência do risco para depressão e de uma elevada frequência de enfermeiros e técnicos de enfermagem classificados com níveis moderados de ansiedade, estes pouco afetaram a qualidade de vida. Isso pode sugerir que estes profissionais podem estar procurando outras formas de promover qualidade de vida para si.

Os casos de ansiedade e risco para depressão leva à reflexão de que esses profissionais estão expostos a uma série de fatores que os predispõem ao adoecimento mental e, diante disso, faz-se necessário que os serviços de saúde elaborem estratégias de promoção e prevenção da saúde mental desses trabalhadores, de forma que o risco para adoecimento mental seja diminuído.

\section{AGRADECIMENTO}

Agradecemos pela participação dos sujeitos na pesquisa.

Todos os autores declararam não haver qualquer potencial conflito de interesses referente a este artigo.

\section{REFERÊNCIAS}

ALMEIDA-BRASIL, C. C. et al. Qualidade de vida e características associadas: aplicação do WHOQOLBREF no contexto da Atenção Primária à Saúde. Ciência \& Saúde Coletiva, v. 22, n. 5, p.1705-1716, 2017.

AMERICAN PSYCHIATRIC ASSOCIATION. Manual diagnóstico e estatístico de transtornos mentais: DSM-5. 5.ed. Porto Alegre: Artmed, 2014.

ARAÚJO, F.D.P. et al. Avaliação da qualidade de vida dos profissionais de enfermagem do atendimento préhospitalar. Rev. Bras. de Medicina do Trabalho, v. 16, n. 3, p. 312-317, 2018.

CAPITÃO, C. G.; TELLO, R. R. Traço e estado de ansiedade em mulheres obesas. Psicologia

hospitalar, v. 2, n. 2, 2004.

COSTA, F. A. Mulher, trabalho e família: os impactos do trabalho na subjetividade da mulher e em suas relações familiares. Pretextos, v. 3, n. 6, 2018. 
COSTA, K. N. F. M. et al. Qualidade de vida relacionada à saúde dos profissionais de enfermagem. Rev enferm UFPE on line, v. 11, p. 881-889, 2017.

GUIMARÃES, A. L. O.; FELLI, V. E. A. Notificação de problemas de saúde em trabalhadores de enfermagem de hospitais universitário. Rev Bras Enferm, v. 69, n. 3, p. 507-14, 2016.

HAUCK FILHO, N.; TEIXEIRA, M. A. P. A estrutura fatorial da Escala CES-D em estudantes universitários brasileiros. Avaliação Psicológica, Porto Alegre, v. 10, n. 1, p. 91-97, maio 2011.

KUREBAYASHI, L. F. S. et al. Auriculoterapia para redução de ansiedade e dor em profissionais de enfermagem: ensaio clínico randomizado. Rev. Latino-Am. Enfermagem, v. 25, 2017.

LEMES, A.G. et al. Estresse e ansiedade em trabalhadores de enfermagem no âmbito hospitalar. Journ. Of Nurs. And Health, v. 5, n. 1, p. 27-37, 2015.

MAHARAJ, S. M.; LEES, T.; LAL, S. Prevalence and Risk Factors of Depression, Anxiety, and Stress in a Cohort of Australian Nurses. Int J Environ Res Public Health, v. 16, n. 1, 2019.

OLIVEIRA, D. M. Afastamento do trabalho por transtornos mentais e comportamentais entre profissionais de enfermagem. Rev Cuid, v. 10, n. 2, p. 1-11, 2019.

PARREIRA, B. D. M. et al. Sintomas de depressão em mulheres rurais: fatores sociodemográficos, econômicos, comportamentais e reprodutivos. Acta Paul Enferm.,, v. 30, n. 4, p. 375-82, 2017.

RIOS, K.A. et al. Evaluation of quality of life and depression in nursing technicians and nursing assistants. Rev. Latino-americana de Enfermagem, v. 18 , n. 3, p. 413-420, 2010.

SANTOS, K. F. R. et al. Aplicabilidade do inventário de Beck nos acadêmicos de enfermagem em uma instituição do sul de Minas Gerais. Enf. em Foco, v. 9, n. 3, p. 81-88, 2018.

SANTOS, M. M.; BARROS, C. R. S.; ANDREOLI, S. B. Fatores associados à depressão em homens e mulheres presos. Rev. bras. epidemiol., v. 22, 2019.

SILVA, D.S.D. et al. Depression and suicide risk among nursing professionals: an integrative review. Rev. da Esc. de Enfermagem da USP, v. 49, n. 6, p. 1023-1031, 2015.
SOUZA, R. F. et al. Repercussões dos fatores associados à qualidade de vida em enfermeiras de unidades de terapia intensiva. Rev. Salud Pública, v. 20, n. 4, p. 453-459, 2018.

TSARAS, K. et al. Predicting Factors of Depression and Anxiety in Mental Health Nurses: A Quantitative Cross-Sectional Study. Orig. Paper, v. 1, n. 72, p. 6267, 2018.

TSUKAMOTO, S. A. S. Violência ocupacional na equipe de enfermagem: prevalência e fatores associados. Acta Paul Enferm., v. 32, n. 4, p. 42532, 2019.

VIEIRA, G.C. et al. Satisfação laboral e a repercussão na qualidade de vida do profissional de enfermagem. Abcs Health Sciences, v. 43, n. 3, p. 186-192, 2018. 